\title{
FLUID FLOWS AROUND TURBOMACHINERY USING AN EXPLICIT PSEUDO-TEMPORAL EULER FEM
}

\author{
N. NIGRO*, M. STORTI† AND S. IDELSOHN† \\ Grupo de Tecnología Mecánica del INTEC CONICET - Universidad Nacional del Litoral Güemes 3450, 3000- \\ Santa Fe, Argentina
}

\section{SUMMARY}

This work is devoted to the simulation by finite elements of nearly incompressible inviscid flows in real 3D geometries, by means of an Euler code based on the SUPG (streamline upwind Petrov-Galerkin) method, explicit forward Euler pseudo-temporal time integration and periodic and absorbing boundary conditions, among other features. The main goal is the application to flow around turbomachinery, with special emphasis on the performance analysis of a given machine, that involves several numerical computations at different operation points. Finally, these results are summarized in the form of characteristic curves.

KEY WORDS SUPG; Petrov-Galerkin methods; finite elements; explicit scheme

\section{INTRODUCTION}

In this work we focus on the numerical solution of incompressible and low subsonic flows around turbomachines like fans, blowers and impellers. A variety of models, ranging from the simplest incompressible potential flow to the compressible Navier-Stokes equations, are applicable, depending on the accuracy required and on the available computing facilities. For inviscid calculations the main possibilities are: potential flow by boundary elements (BEM) or panel methods, or direct solution of the Euler equations by finite differences (FDM) or finite elements (FEM). Potential flow by BEM is, by and large, the most efficient method, and its results are correct as long as the flow characteristics to be studied are not too sensitive to the position of the wake. ${ }^{1-4}$ If this is so, the BEM allows for a very fast computation of pressure distribution and aerodynamic forces, which is enough, in most cases, for a preliminary design. This is done assuming a prescribed position of the wake, and a linear system of equations is obtained. For improved modelling of the wake, its surface must be also covered with panels, and its position must be adjusted in order to satisfy the equality of pressures in both sides of the wake. These restrictions are non-linear in nature and the efficiency of the method deteriorates as compared to an FEM solution of the Euler equations. In addition, a much broader range of complex phenomena such as inlet swirl and compressibility effects can be included with this last model, so that it becomes a useful complementary study in the last stage of design.

\footnotetext{
${ }^{*}$ Research Fellow from CONICET

†CONICET Research Staff Members

CCC 0748-8025/95/030199-13

(C) 1995 by John Wiley \& Sons, Ltd.
} 
The algorithm we present here is based on the SUPG method applied to the Euler equations in a conservative form. ${ }^{5-9}$ The SUPG method adds the appropriate amount of numerical diffusivity to avoid oscillations, characteristic of advective systems but in a context of a weighted residual method, which guarantees consistency. A new expression for the matrix of intrinsic time scales, characteristic of SUPG schemes, is proposed, which allows for a closed expression for elements of arbitrary aspect ratios and shear. The resulting non-linear system of equations is solved in pseudo-temporal form by a standard forward-Euler scheme. A local time step and absorbing boundary conditions are used to accelerate the convergence to the steady state, ${ }^{5}$ and periodical boundary conditions allow the computations to be carried on one representative sector only, even if no planes of symmetry are present.

\section{GOVERNING EQUATIONS}

The governing equations for an inviscid fluid in a non-inertial frame, rotating with constant angular velocity $\mathbf{\Omega}$, in conservative form, are

$$
\mathbf{u}, t+\mathbf{f}_{a, i}^{i}=\mathbf{b}
$$

where $\mathbf{u}=[\rho, \rho \mathbf{v}, \rho e]^{\mathrm{T}}$ is the state vector of the fluid, $\rho$ is the density, $\mathbf{v}$ the velocity vector and $e$ the total energy,
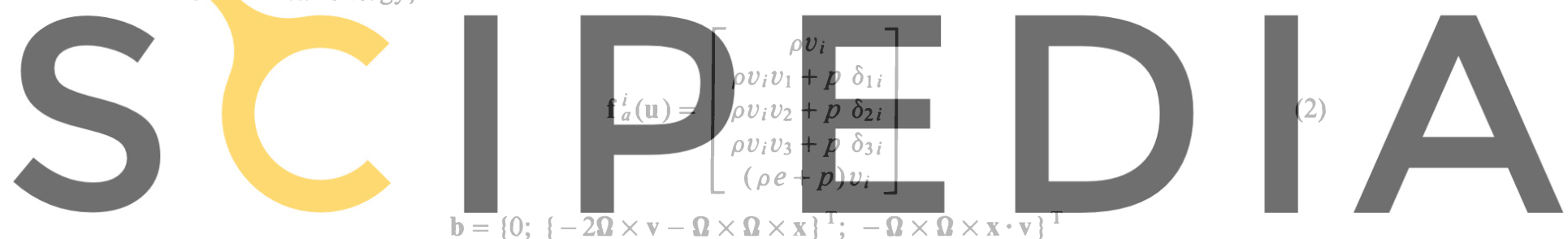

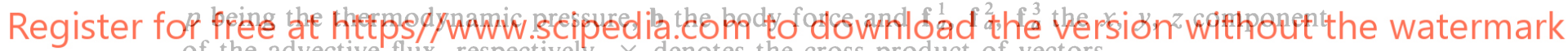
of the advective flux, respectively. $X$ denotes the cross product of vectors.

As is usual for incompressible fluids, the centrifugal force can be absorbed as a potential body force in the static pressure, simplifying the source term. Finalily, these equations are closed with the state equations for the gas and the energy: $\theta=\theta(p, \rho), e=e(p, \rho)$.

\section{DISCRETIZATION SCHEME}

The system (1) is advective-reactive, which means that it does not contain any diffusive terms. As is well known, the application of the standard Galerkin (centred) technique to purely advective equations, as the present case is, leads inevitably to spurious oscillations which pollute the solution. For advective diffusive systems the oscillations can be suppressed by straightforward refinement of the mesh, at the expense of the increase in computing time. This strategy becomes more and more expensive as the flows become advection-dominated, and unapplicable to purely advective flow.

Hence, a family of schemes to avoid these oscillations has been developed by many authors. In the context of the FEM, SUPG is one of the most popular schemes, since it does not contain free parameters at all (at least in the advection-dominated limit), it adds the correct amount of numerical diffusion to avoid oscillations, and, more importantly, it can be cast as a weighted residual method and, then, consistency is assured. ${ }^{6-8}$ 


\subsection{Spatial discretization}

In the weighted residual form of the SUPG formulation the weighting functions are modified by the addition of $C^{-1}$ perturbations. The Euler-Lagrange form applied to the compressible Euler equations yields the following expression:

$\mathbf{0}=\sum_{\mathrm{e}} \int_{\Omega^{\mathrm{e}}}(\mathbf{N}+\mathbf{P})\left(\mathbf{u}_{, t}+\mathbf{f}_{a, i}^{i}-\mathbf{b}\right) \mathrm{d} \Omega-\int_{\Gamma_{\text {slip }}} \mathbf{N \mathbf { f } _ { n } ^ { ( 2 ) }} \mathrm{d} \Gamma-\int_{\Gamma_{\text {int }}} \mathbf{N} \llbracket \mathbf{f}_{n} \rrbracket \mathrm{d} \Gamma \quad \forall N_{i}$ in $V^{i}$

with the unknown variable vector $\mathbf{u}$ and the conservative fluxes $\mathbf{f}_{a}^{i}$ interpolated by the standard piece-wise-bilinear form functions $\mathbf{N}=\operatorname{diag}\left\{N_{1}, \ldots, N_{m}\right\}$, with $V^{i}$ a typical $C^{0}$ finite element space, and $m$ being the number of degrees of freedom at each node. Therefore, it is verified that the flux projection jump on the normal direction over the interelement boundaries should be neglected $\left(\llbracket \mathfrak{f}^{n} \rrbracket=0\right)$. On the other hand, the slip boundaries contribution term takes into account only a part of the conservative fluxes given by $\mathbb{f}_{n}^{(2)}=\left\{v_{n} \rho ; v_{n} \rho \mathbf{v} ; v_{n} \rho e\right\}^{\mathrm{T}}$

\subsection{The matrix of intrinsic time scales}

Consider, first, the case of the scalar advection equation:

$$
\mathrm{v} \cdot \nabla \phi=b
$$
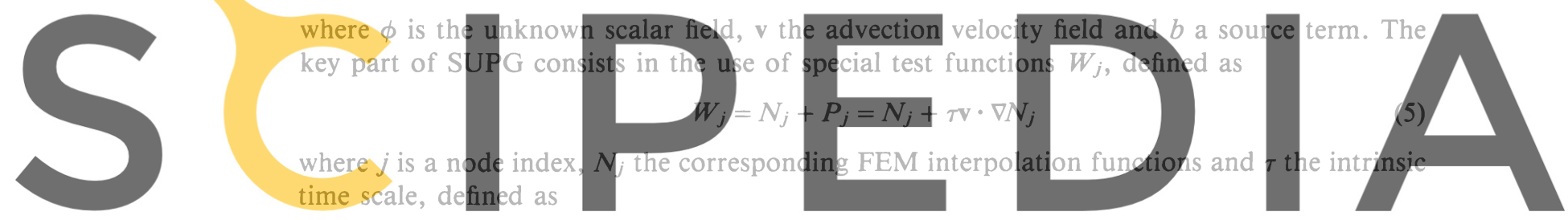

where $\xi(\mathbb{x})$ is the transformation of the element into the 'master' element: $-1 \leqslant \xi$, $\eta \leqslant 1$.
For systems of equations $\tau$ is a matrix and the natural extension of $(6)$ has been proposed by Mallet et $a l^{7,9}$ and is

$$
\boldsymbol{\tau}=|\tilde{\mathbf{A}}|^{-1}
$$

where $\tilde{\mathbf{A}}_{j}=\left(\partial \xi_{j} / \partial x_{i}\right) \mathbf{A}_{i}$ and $\mathbf{A}_{i}$ are the Jacobians of the fluxes: $\mathbf{A}_{i}=\partial \mathbf{f}_{a}^{i} / \partial \mathbf{u} .|\tilde{\mathbf{A}}|_{2}$ is the $L_{2}$ norm of the vector matrix defined as

$$
|\tilde{\mathbf{A}}|_{2}=\sqrt{ }\left(\tilde{\mathbf{A}}_{x}^{2}+\tilde{\mathbf{A}}_{y}^{2}+\tilde{\mathbf{A}}_{z}^{2}\right)
$$

The non-trivial part of the calculation consists in the evaluation of the square root of a matrix. In general, the rule is to find a change of basis matrix to bring the argument matrix to its diagonal form, by solving an eigenvalue problem, computing the square root of the diagonal terms and coming back to the original basis. However, for $3 \mathrm{D}$ problems one is faced with a five degree equation, which must be solved by a numerical routine, and the CPU time becomes excessively high. For cubic elements there exists a transformation that reduces the determinantal equation order to a quadratic one, and then the problem is solvable in a closed form. ${ }^{9}$ For low-aspect-ratio, mildly sheared elements, an approximate expression for the matrix of intrinsic time scales can be obtained by replacing the actual element by a cubic one with approximately the same dimensions, but the resulting scheme is too diffusive for high 
aspect ratios. This is termed by Mallet as the 'Cartesian approximation'. Furthermore, this high diffusion imposes a very low time step and the global CPU time is not reduced. Such highaspect-ratio elements are common in $3 \mathrm{D}$ meshes around turbomachines as the variation of the unknowns in the span-wise direction (i.e. along the axis of the blade) is small and it allows for the use of very elongated elements in this direction. On the other hand, variations in the other directions (stream-wise and normal) are too strong, especially at the leading and trailing edges. Aspect ratios as high as $1: 100$ are common in those places.

In order to solve this problem, we propose to compute the norm of the Jacobian vector in the $L_{1}$ norm, i.e.

$$
\boldsymbol{\tau}=|\tilde{\mathbf{A}}|_{1}^{-1}
$$

with

$$
|\tilde{\mathbf{A}}|_{1}=\left|\tilde{\mathbf{A}_{x}}\right|+\left|\tilde{\mathbf{A}}_{y}\right|+\left|\tilde{\mathbf{A}}_{z}\right|
$$

Now, each one of the three terms in the right-hand side can be calculated in closed form, since the involved matrices $\tilde{\mathbf{A}}_{i}$ are projections of the flux-Jacobian vectors. With this matrix of intrinsic time seales, elements with arbitrary aspect ratio can be used.
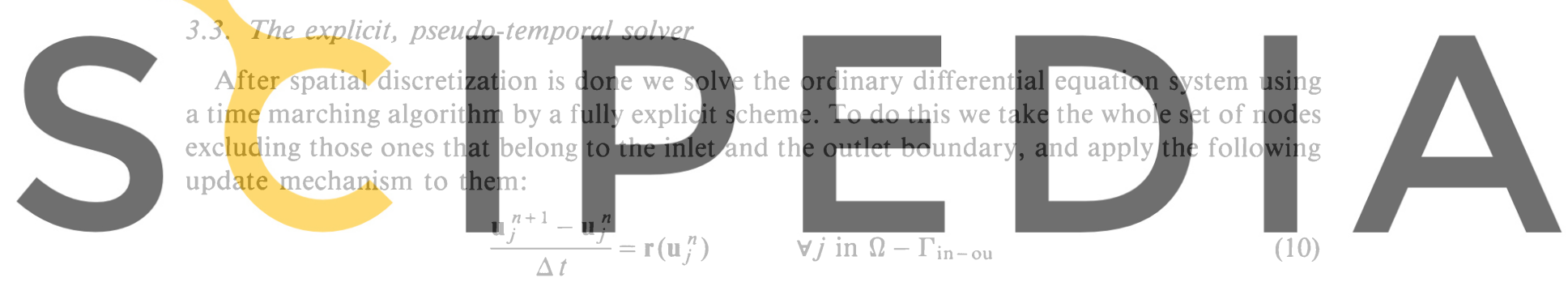

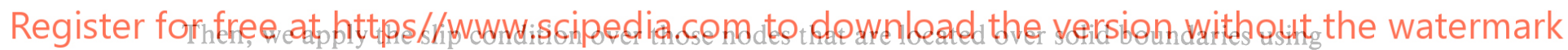
the following projection:

$$
\tilde{\mathbf{v}}_{j}^{n+1}=\mathbf{v}_{j}^{n+1}-\left(\mathbf{v}_{j}^{n+1} \cdot \mathbf{n}_{j}\right) \mathbf{n}_{j} \quad \forall j \text { in } \Gamma_{\text {slip }}
$$

For those nodes that belong to the inlet and the outlet we apply other kind of update given by:

$$
\frac{\mathbf{u}_{j}^{n+1}-\mathbf{u}_{j}^{n}}{\Delta t}=\Pi^{-} \mathbf{r}\left(\mathbf{u}_{j}^{n}\right) \quad \forall j \text { in } \Gamma_{\text {in-ou }}
$$

with $\boldsymbol{\Pi}^{-} \mathbf{u}=\left(\mathbf{S} \tilde{\Pi}^{-} \mathbf{S}^{-1}\right) \mathbf{u} ; \tilde{\boldsymbol{\Pi}}^{-}=\operatorname{diag}\{0, \ldots, 0,1, \ldots, 1\}$, where the first $l$ zeros correspond to positive eigenvalues (outlet modes) and the other $(m-l)$ zeros to the negative eigenvalues (inlet modes).

To impose periodic boundary conditions, the residual is computed by summing all contributions coming from elements in a reduced set of elements composed of a representative sector $\mathrm{ABCD}$ and an additional layer of elements CEFD (see Figure 1). The representative sector is such that nodes $a$ and $b$ on corresponding sides of the sector are equivalent, since they are obtained through a rotation of $2 \pi / n_{\mathrm{bl}}$. The additional layer (marked as hatched in the Figure) is added to the computation, in such a way that the residuals are computed correctly for the 'internal nodes' (as $b$ and $g$ ), since all elements surrounding them are included in the summation. After the update (10) is performed for all internal nodes the state vector for the 'external nodes' as $a$ and $f$ are computed from appropriated symmetry operations on the 


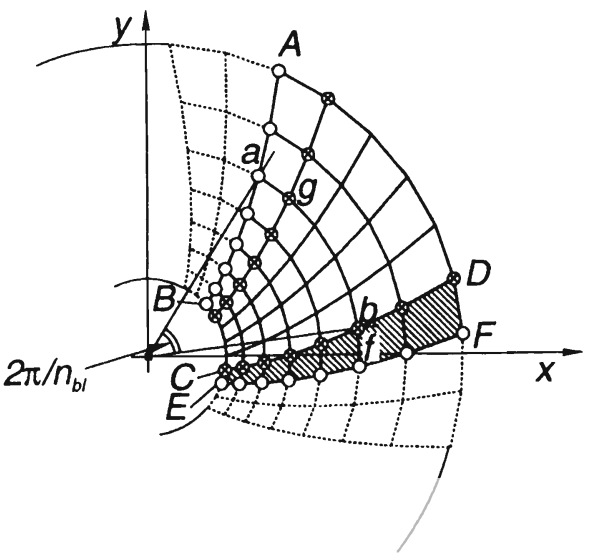

Figure 1. Periodical boundary conditions: elements in representative sector plotted with solid lines; additional layer of elements has been hatched; the rest of the elements (that are not actually involved in any computations) are shown in dotted lines: at update time, state vector for assigned nodes $(0)$ is set equal to that on corresponding node $(\times)$, after applying proper transformation (see equation (13))
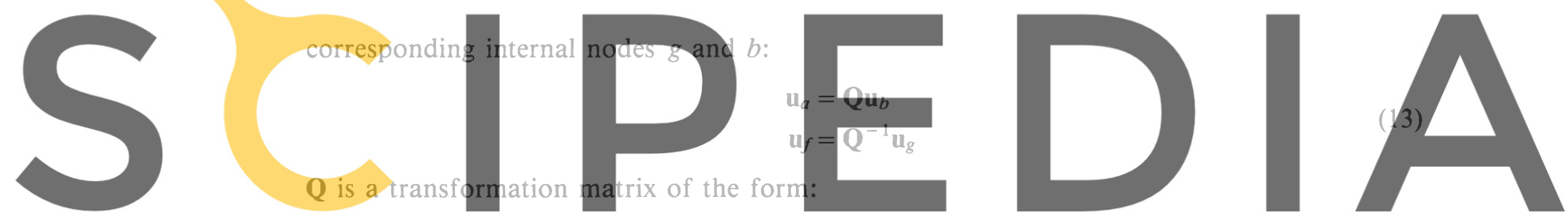

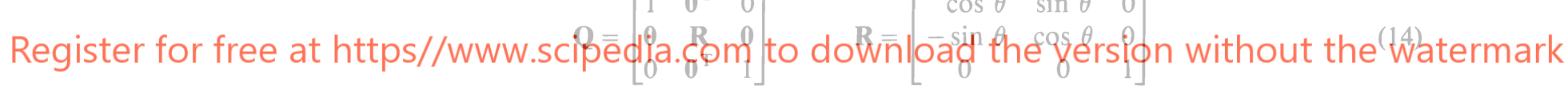

$\mathbb{R}$ is a rotation matrix of $\theta=2 \pi / n b l$ radians with respect to the $z$-axis. The particular form of $\mathbf{Q}$ is due to the tensorial character of the different components of the state vector $\mathrm{u}: \rho$ and $\rho e$ transform as scalars, whereas $\rho \mathbf{v}$ transforms as a vector. Although the explanation has been given for a structured mesh, as that shown in Figure 1, it can be extended easily to completely non-structured meshes.

\section{NUMERICAL EXAMPLES}

\subsection{Geometry and mesh description}

The rotor has $n_{\mathrm{bl}}=7$ blades; internal/external radius: $R_{\mathrm{int}}=0.094 \mathrm{~m} ; R_{\mathrm{ext}}=0.310 \mathrm{~m}$. The chord, pitch and shape of the blades vary in the radial direction (see Figures 2-4). The FEM mesh around one representative sector has 4816 elements and 6057 nodes (see Figures 5-8). It is a structured mesh composed of eight layers of 602 elements in the radial sense. Each layer is essentially a ' $\mathrm{C}$ ' mesh of 10 (normal to the blade) layers of 58 elements (tangential). As a whole, there are 384 elements covering the blade surface. Note that for a similar calculation without periodic conditions the number of elements is raised to $58 \times 10 \times 8 \times 7=32,480$. 


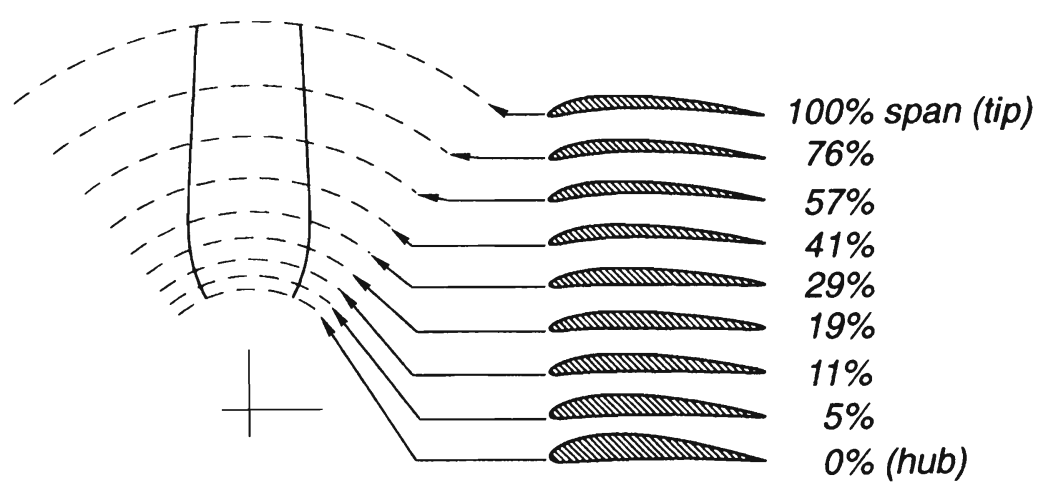

Figure 2. Section shape at various stations: value at right of aerofoil is the span $(0 \%$ at hub, $100 \%$ at tip)
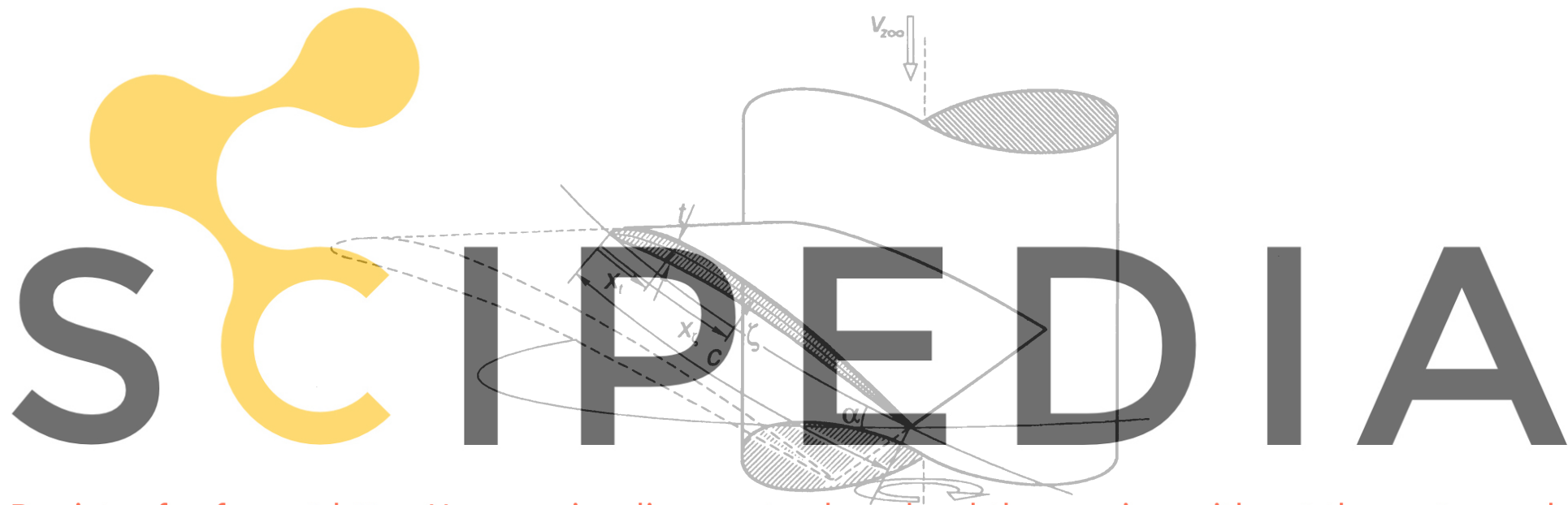

Register for free at https//www.scipedia.com to downloadathe version without the watermark

Figure 3. Definition of aerofoil geometrical parameters as maximum thickness $t$, maximum camber $\zeta$ and respective

locations $x_{t}, x_{5}$ (all in $\%$ of chord, locations from LE), chord $c$ and incidence angle $\alpha$ measured with respect to rotation plane

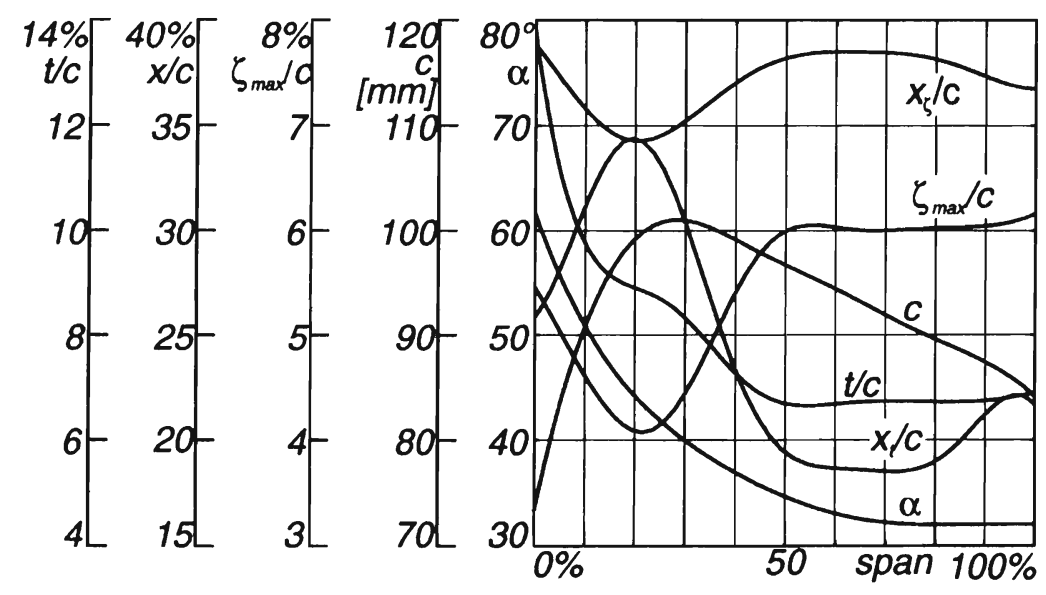

Figure 4. Aerofoil section parameters as function of span 

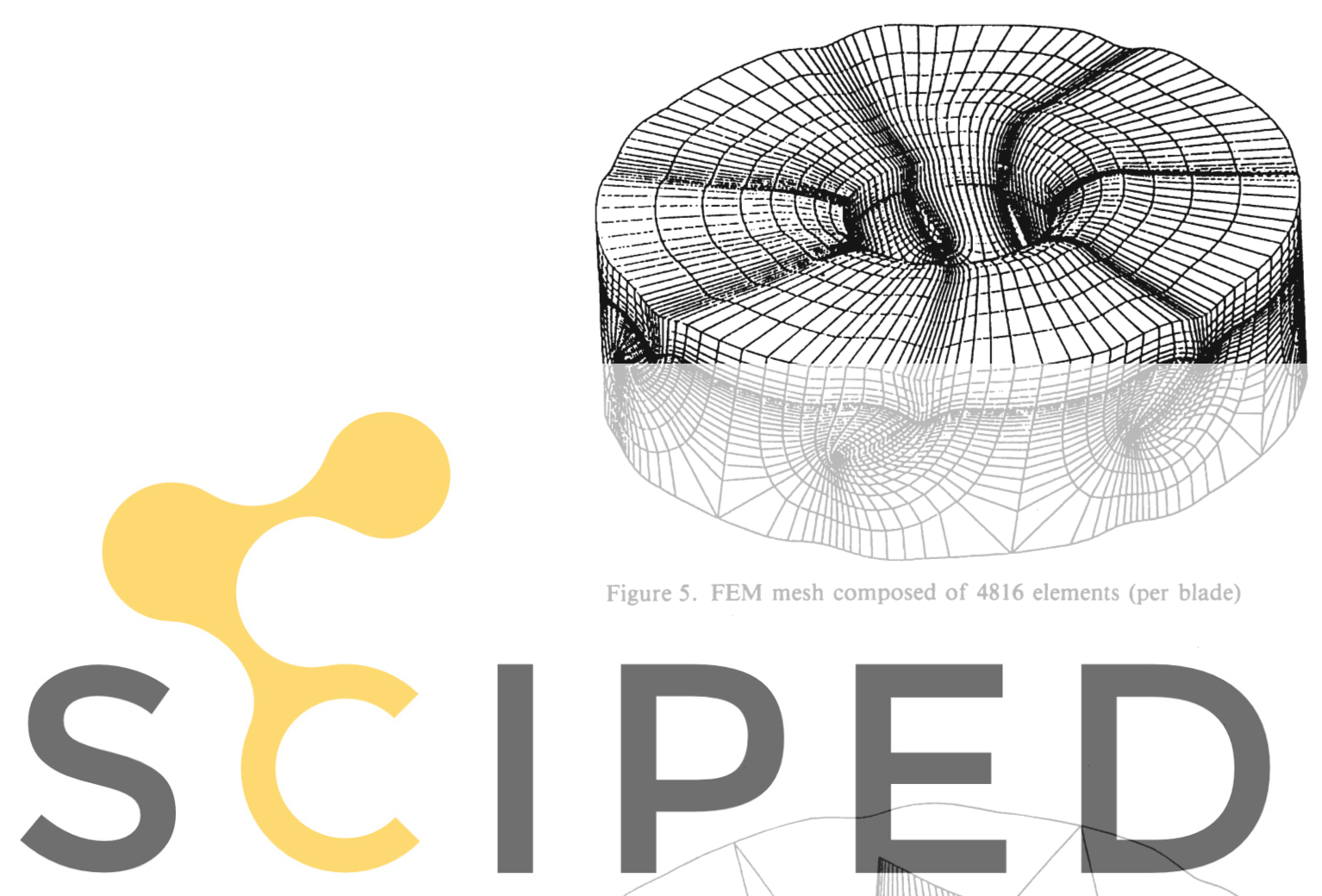

Figure 5. FEM mesh composed of 4816 elements (per blade)

\section{Register for free at https//www.scipedia.com to downtbad the vers ow without the watermark}

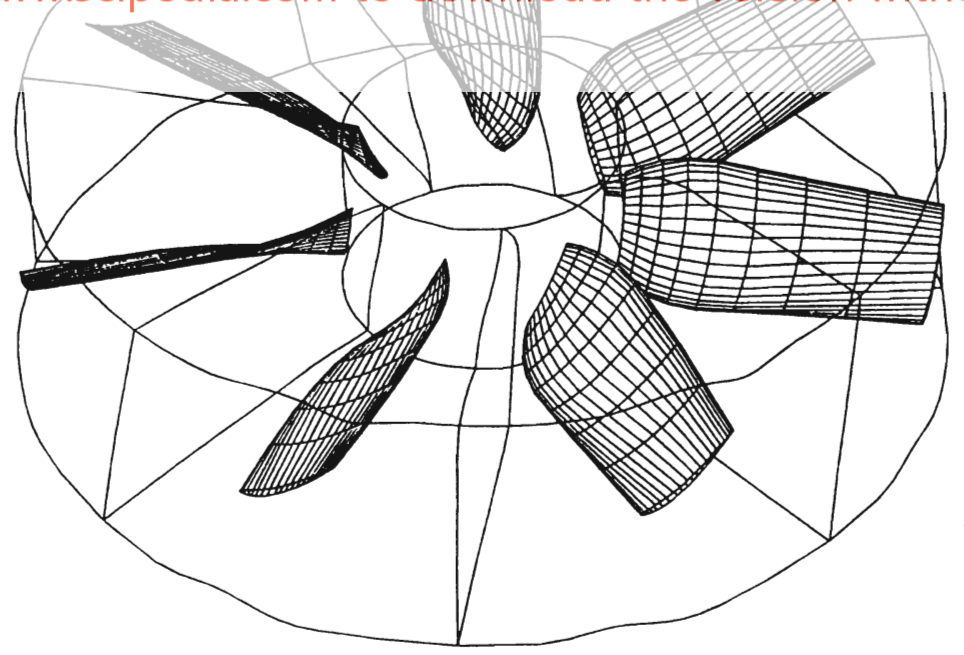

Figure 6. View of element faces covering blade surfaces (384 elements per blade) 


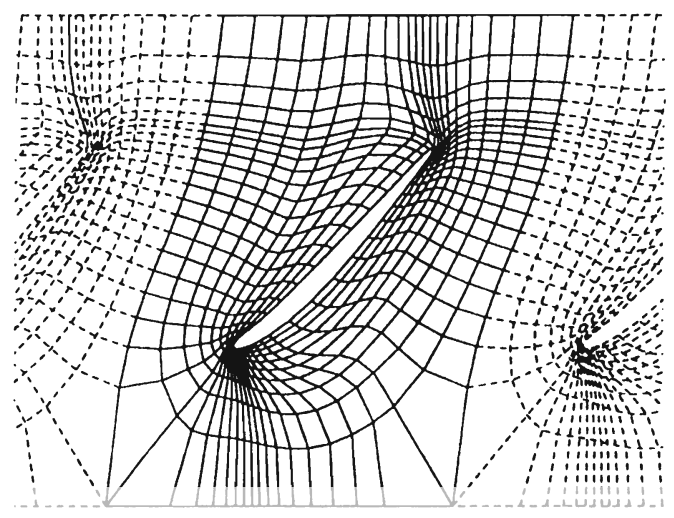

Figure 7. 'C' mesh around plane cascade at $r=136 \mathrm{~mm}$ (29\% span)

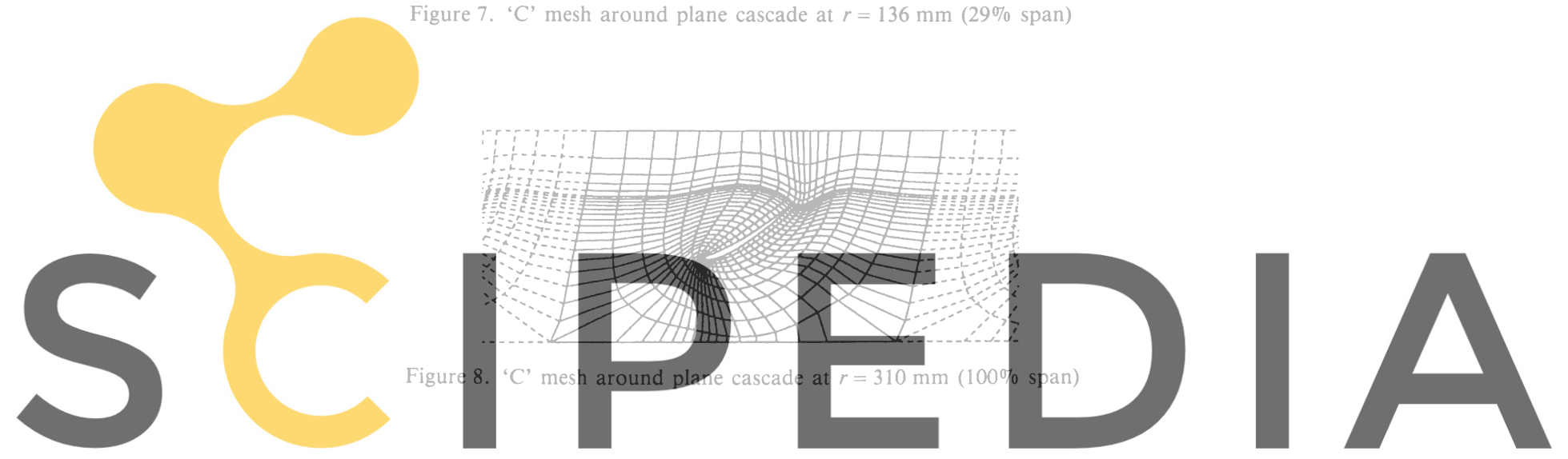

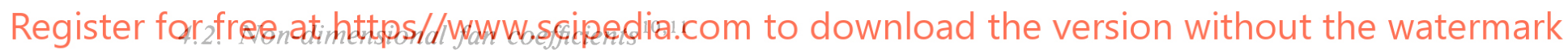

All cases were run at a fixed speed of $\Omega=103 \cdot 25 \mathrm{rad} / \mathrm{s}(n=985 \mathrm{rev} / \mathrm{min})$. The flow rate $Q$ was varied from mean axial velocities of $v_{z}=7.43 \mathrm{~m} / \mathrm{s}$ to $20 \mathrm{~m} / \mathrm{s}$. In fact, the oniy nondimensional number characterizing fan operation is the capacity coefficient $C_{\mathrm{Q}}=Q /\left(n D^{3}\right)$, where $D$ is the external diameter, and the above mean axial velocities correspond to $0 \cdot 5<C_{\mathrm{Q}}<1 \cdot 5$. From the pressure field obtained in the FEM analysis, the total torque $M$ and the total axial force $F_{z}$ can be computed as

$$
F_{z}=n_{\mathrm{b} 1} \int_{\mathrm{S}}\left(-p n_{z}\right) \mathrm{d} s \quad M=n_{\mathrm{b} 1} \int_{\mathrm{S}}\left(-p n_{\theta}\right) r \mathrm{~d} s
$$

where $n_{\mathrm{z}}, n_{\theta}$ are axial and circumferential components of the unit outward normal vector to the blade surface $\mathrm{S}$. The jump in static pressure $\Delta p_{\text {st }}$ across the fan is computed by means of a global balance of axial momentum, and then the static head coefficient is computed as

$$
C_{\mathrm{H}}=\frac{\Delta p_{\mathrm{st}}}{\rho n^{2} D^{2}}
$$

The total shaft power is $P=M \Omega$ and the corresponding non-dimensional coefficient is

$$
C_{\mathrm{P}}=\frac{P}{\rho n^{3} D^{5}}
$$


The useful power (also called air power) is given by $Q \Delta p_{\text {st }}$ and the static efficiency is

$$
\eta_{\mathrm{st}}=\frac{P_{\mathrm{air}, \mathrm{st}}}{P}=\frac{C_{\mathrm{Q}} C_{\mathrm{H}}}{C_{\mathrm{P}}}
$$

\subsection{Numerical results}

The accuracy of an Euler code depends, mainly, on its ability to obtain (spatially) stable solutions without adding excessive numerical diffusion. The magnitude of this artificial viscous force can be estimated through the balance of axial forces, and it was estimated to be less than $1 / 1000$ of the axial force on the rotor.

In Figure 9 we can see the $C_{\mathrm{p}}$ curves for all sections at an average axial flow velocity of 18 $\mathrm{m} / \mathrm{s}\left(C_{\mathrm{Q}}=1 \cdot 26\right)$, near wide-open conditions.

Furthermore, we computed the axial velocity, averaged over the circumferential direction, for the inlet and outlet sections, as

$$
V_{z}(r)=\frac{1}{2 \pi} \int_{\phi=0}^{2 \pi} v_{z}(r, \phi) \mathrm{d} \phi
$$

The radial distribution of axial velocity is shown for several operating conditions $\left(C_{Q}\right)$ in

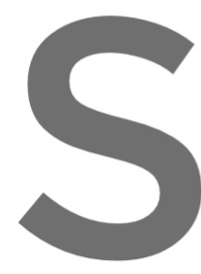

Figures 10 a

behayiour is

stronger for
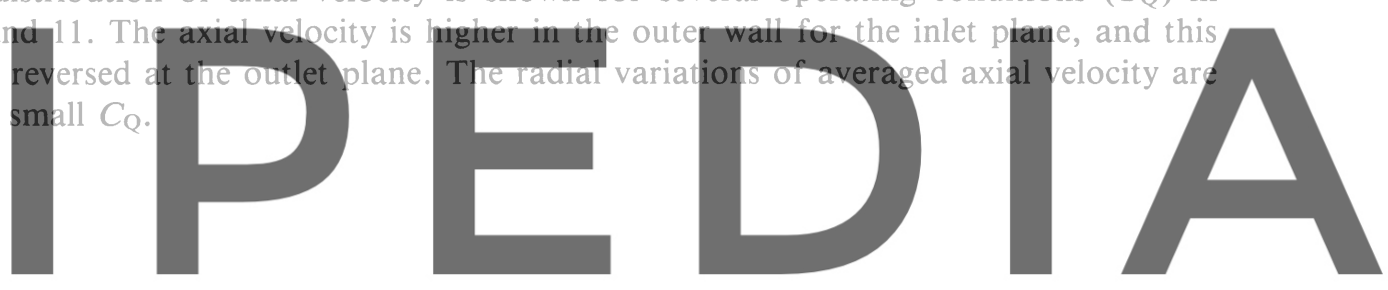

Register for free at https//www.Sclipedia.com to download the version without the watermark

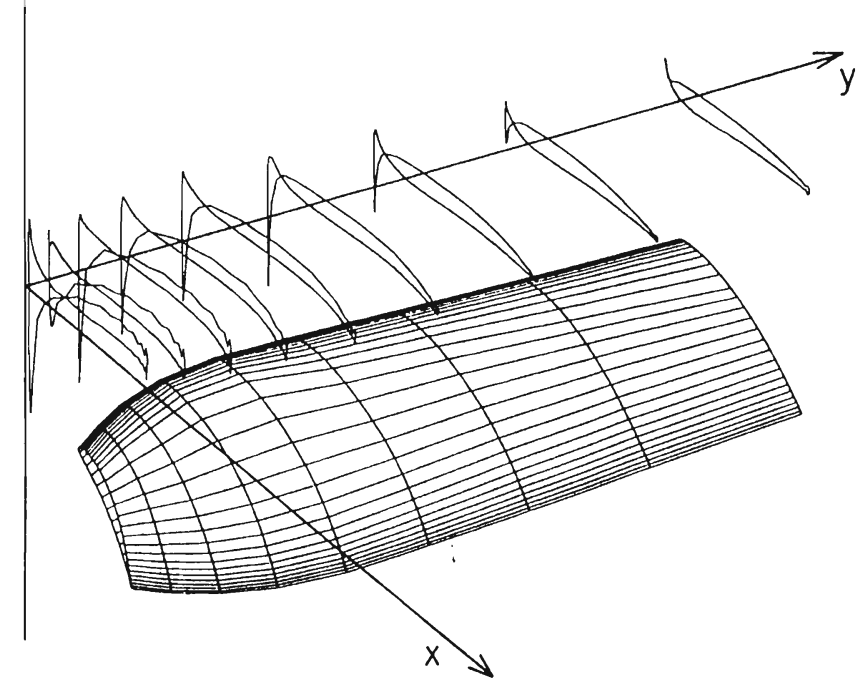

Figure 9. $C_{\mathrm{p}}$ distribution on blade at $\left\langle v_{z}\right\rangle=18 \mathrm{~m} / \mathrm{s}\left(C_{\mathrm{Q}}=1 \cdot 26\right)$ 


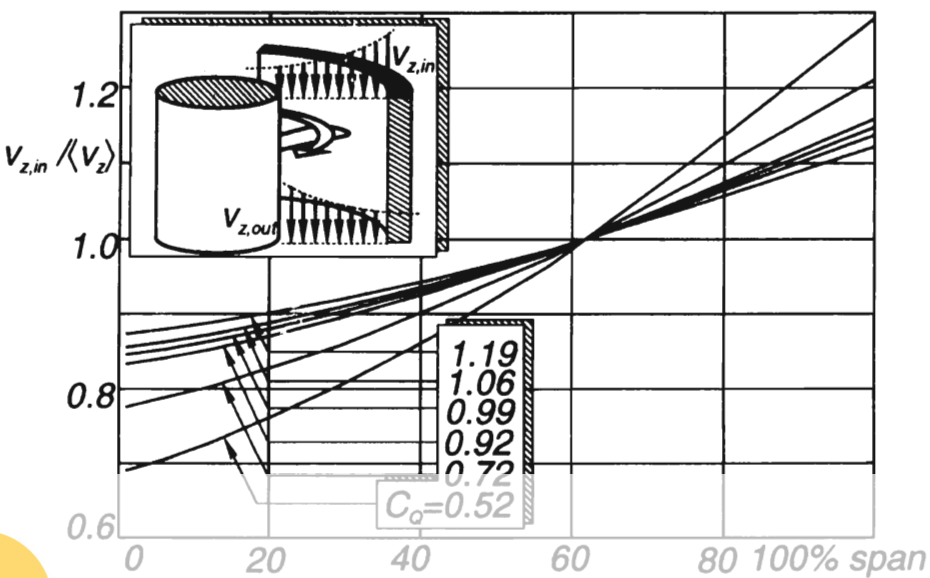

Figure 10. Circumferentially averaged axial velocities as function of span at the inlet plane, for several operating conditions ( $C_{\mathrm{Q}}$ indicated for each curve on right margin)

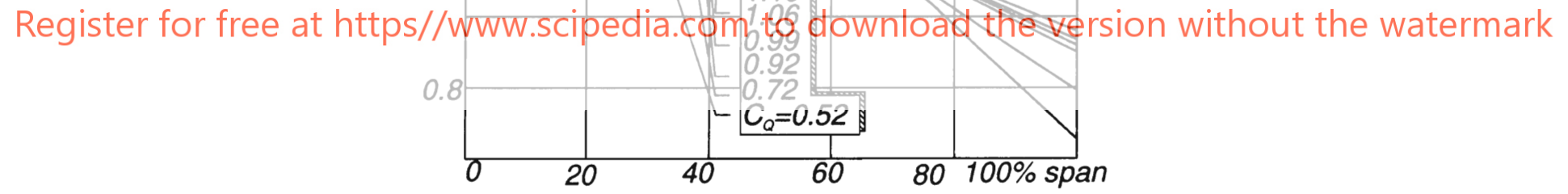

Figure 11. Circumferentially averaged axial velocities at outlet plane

We compute also the radial distribution of axial and circumferential force as

$$
\begin{aligned}
& f_{z}(r)=\int_{\mathscr{C}(r)}\left(-p n_{z}\right) \mathrm{d} l \\
& f_{\theta}(r)=\int_{\mathscr{C}(r)}\left(-p n_{\theta}\right) \mathrm{d} l
\end{aligned}
$$

where the integral is evaluated over the profile section $\mathscr{C}(r)$, and $n_{\theta}, n_{z}$ are the circumferential and axial components of the unit vector $\hat{\mathbf{n}}$, normal to the blade surface. These quantities have been plotted against $r / D$ in Figures 12 and 13. The global axial force and torque are related 


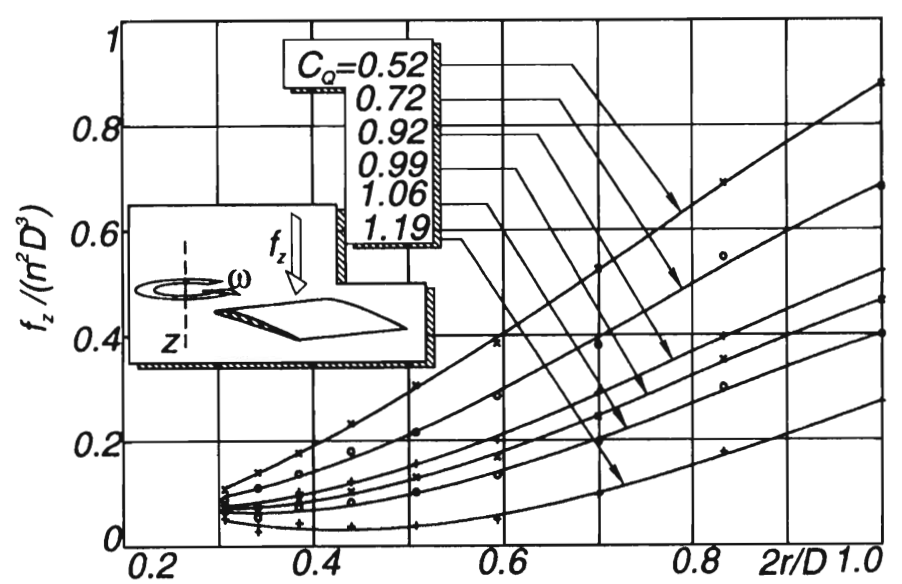

Figure 12. Axial force per unit span at several operating conditions

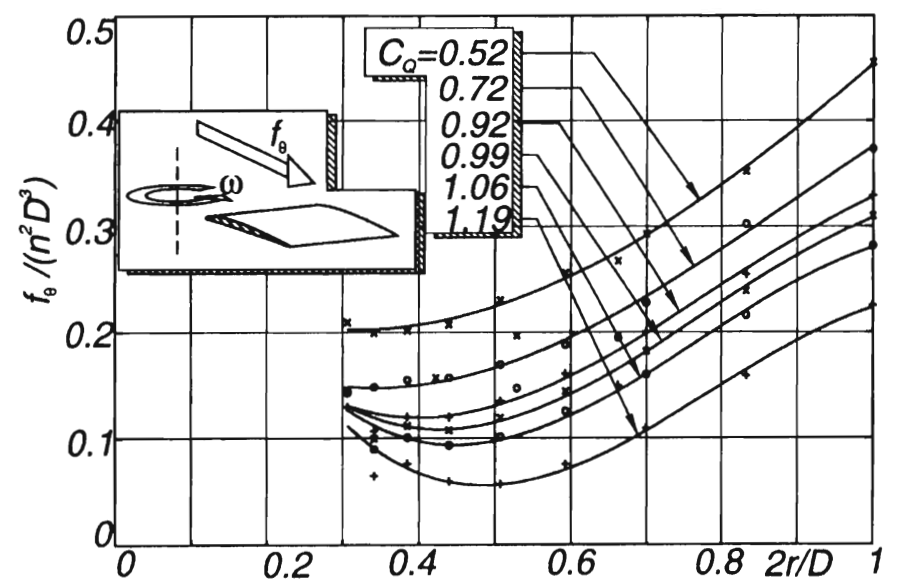

Figure 13. Circumferential force per unit span at several operating conditions

to them as:

$$
\begin{aligned}
& F_{z}=n_{\mathrm{bl}} \int_{R_{\mathrm{int}}}^{R_{\mathrm{ex} \mathrm{l}}} f_{z}(r) \mathrm{d} r \\
& M=n_{\mathrm{bl}} \int_{R_{\mathrm{int}}}^{R_{\mathrm{ext}}} f_{\theta}(r) r \mathrm{~d} r
\end{aligned}
$$

The most important contribution (and also to static head and power) comes from the external region, as usual, with an almost linear variation with radius for $f_{z}$.

\subsection{Fan characteristic curves}

The non-dimensional coefficients of Section 4.2 are shown in Figure 14. Both power and head are monotonically decreasing with flow rate. The head becomes zero for $C_{\mathrm{Q}}=1 \cdot 3$, 


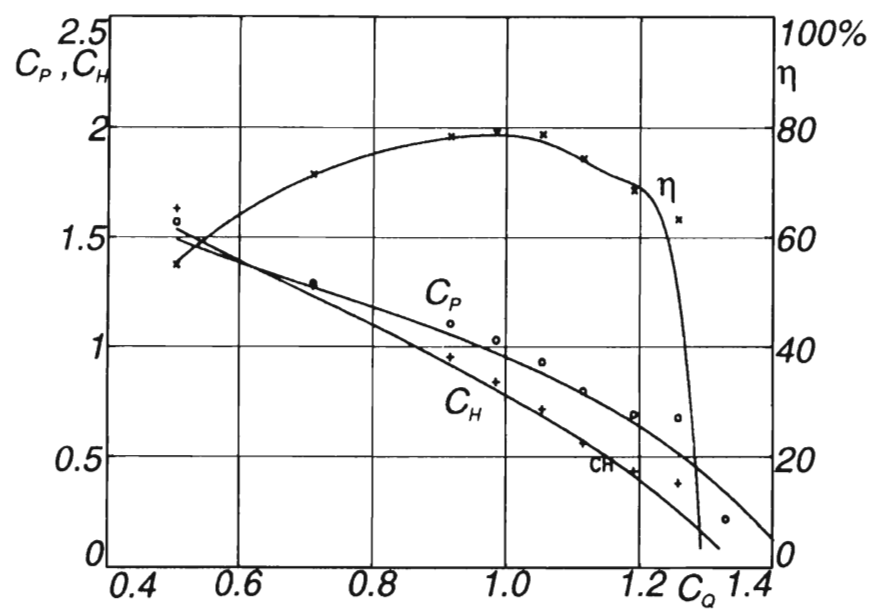

Figure 14. Fan characteristic curves: non-dimensional power $C_{\mathrm{P}}$, static head $\left(C_{\mathrm{H}}\right)$ coefficients and efficiency $\eta$ as function of capacity coefficient $C_{\mathrm{Q}}$

corresponding to 'wide-open' operating conditions, with a small power delivery. This situation corresponds to an angle of $28^{\circ}$ between the undisturbed (relative) streamline and the circumferential direction at the tip section, whereas the incidence of the mean chord at that section is $32^{\circ}$.

Efficiency exhibits a rather smooth peak at $C_{\mathrm{Q}} \approx 1$. A characteristic feature of axial fans is a rather abrupt change of sign in the slope for lower flow rates due to stalling at high incidence angles. This is a purely viscous phenomenon and cannot be directly predicted from the Euler code, but the point of separation can be estimated, with reasonable accuracy, through a secondary boundary layer calculation. Viscous losses can also be estimated in this way.

\section{CONCLUSIONS}

We have presented the well known SUPG finite element formulation applied to turbomachinery simulation. The computational cost optimization is one of the most important subjects to study due to the high number of degrees of freedom appearing in this kind of problem. We have included in this work several interesting features to accelerate the convergence, like those of absorbing and periodical boundary conditions and a less expensive way to calculate the matrix of intrinsic time scales for the SUPG operator. Thanks to these, it was possible to make a whole study of the machine performance at different operation points in a reasonable time using PC-based systems. We have included the most important results and characteristic curves obtained with the developed code.

\section{ACKNOWLEDGEMENT}

The authors wish to express their gratitude to Consejo Nacional de Investigaciones Científicas y Técnicas (CONICET, Argentina) for its financial support. 


\section{REFERENCES}

1. C. Y. Hsin, 'Development and analysis of panel methods for propellers in unsteady flows', $\mathrm{PhD}$ thesis, Massachussets Institute of Technology, Department of Ocean Engineering, Cambridge, Massachussets, 1991.

2. S. A. Kinnas and C. Y. Hsin, 'Boundary element method for the analysis of the unsteady flow around extreme propeller geometries', AIAA J., 30, 688-696 (1992).

3. T. Maître, 'Modelisation de l'ecoulémént autour d'une hélice marine par la méthode du potentiel', Ph.D thesis, Institut National Polytechnique de Grenoble, 1988.

4. M. Gennaretti and L. Morino, 'A boundary element method for the potential, compressible aerodynamics of bodies in arbitrary motion', Aer. J., 96, 15-19 (1992).

5. C. Baumann, M. Storti and S. Idelsohn, 'A Petrov-Galerkin technique for the solution of transonic and supersonic flows', Comput. Methods Appl. Mech. Eng., 95, 49-70 (1992).

6. T. J. R. Hughes and T. E. Tezduyar, 'Finite element methods for first-order hyperbolic systems with particular emphasis on the compressible Euler equations', Comput. Methods Appl. Mech. Eng., 45, 217-284 (1984).

7. T. J. R. Hughes and M. Mallet, 'A new finite element method for CFD: III. The generalized streamline operator for multidimensional advection-diffusion systems', Comput. Methods Appl. Mech. Eng., 58, 305-328 (1986).

8. T. J. R. Hughes and M. Mallet, 'A new finite element method for CFD: IV. A discontinuitycapturing operator for multidimensional advective-diffusive systems', Comput. Methods Appl. Mech. Eng., 58, 329-336 (1986).

9. M. Mallet, 'A finite element method for CFD', Ph.D thesis, Stanford University, 1985.

10. B. Eck, Fans. Design and operation of centrifugal, axial-flow and cross-flow fans, Pergamon Press, 1973.

11. W. C. Osborne, Fans, Pergamon Press, 1977.

12. G. Moretti, 'Orthogonal grids around difficult bodies', AIAA J., 30(4), 933-938 (1992).

13. M. Storti, 'Mesh generation around a 3D rotor geometry', ${ }^{\mathrm{G}} \mathrm{T}_{\mathrm{M}}$ Internal Report 3/93, 1993 (in Spanish).

14. T. Baumeister, 'Turbomachinery', in Handbook of Fluid Dynamics (ed. V. C. Streeter), Chap. XIX, McGraw-Hill, New York, 1961. 